\title{
Forging synergies in drug discovery
}

\author{
The pharmaceutical industry faces major challenges in delivering the next generation of therapeutic agents. The shared \\ interests of pharmaceutical researchers and chemical biologists provide impetus for new drug discovery innovations.
}

$T^{\mathrm{h}}$ he pharmaceutical industry, known for its leadership in scientific innovation and as a darling of investors, is currently encountering serious challenges. Increasing costs, a limited drug pipeline, and the impending loss of patent coverage for 'blockbuster' drugs are changing the landscape of pharmaceutical science. Though business leadership will be essential to overcome these challenges, the fundamental problem - that traditional models of drug discovery and development are not delivering an adequate supply of drugs to meet medical needs and to sustain the industry - remains unresolved. New scientific approaches will be required to build a strong foundation for the future of drug discovery research. Chemical biology is uniquely positioned to contribute to this arena.

Pharmaceutical companies have a strong track record in identifying and developing small-molecule compounds for therapeutic applications despite an inherently low success rate. Because of the substantial investments required to produce a relatively small product portfolio, the business models of the pharmaceutical sector are fundamentally different from those of more traditional businesses. Yet these different models are subject to the same market forces. Such pressures may have fueled the industry's emphasis on 'blockbuster' drugs - therapeutic agents of significant medical importance that make up the bulk of annual revenues for pharmaceutical companies. In parallel with other industries, past successes and market pressures have led to mergers that have consolidated expertise and resources in a smaller number of large pharmaceutical companies.

Following a record of success, the pharmaceutical industry has now reached a critical stage. Many of the blockbuster drugs that appeared on the market in the late 1990s will lose patent coverage in the coming years and will be replaced by cheaper generic versions (B. Martinez and J. Goldstein, "Big pharma faces grim prognosis," The Wall Street Journal, 6 December 2007, A1). In parallel, the pipeline of new drugs appears incapable of generating replacements for today's top performers. Recent analyses indicate that despite a more than doubling of global pharmaceutical research and development budgets in the past decade, the number of drugs approved by the US Food and Drug Administration has dropped from 24 in 1998 to 13 in 2006 ("Beyond the pill," The Economist, 25 October 2007). In addition, pharmaceutical companies are facing mounting pressure to accede to price controls and compulsory licensing to support broader access to medical treatments ("A gathering storm," The Economist, 7 June 2007). Many critics feel that the pharmaceutical industry, in response to these pressures, has become overly cautious and focused on marketing and 'me too' drugs at the expense of research and development priorities, a strategy that has exacerbated the lack of a strong drug pipeline.

Overcoming these current challenges will require concerted business and scientific leadership. While management decisions may limit financial losses in the short term, such actions have already affected large num- bers of scientists who have been laid off by pharmaceutical companies in the past year (R. Mullin, “New Novartis cuts target 2,500 jobs," Chemical and Engineering News, 24 December 2007, 8). Chemists, who are essential to small-molecule drug discovery and development, have felt these effects more than their biologist colleagues due to an increased emphasis on biologic therapies (A. Johnson, "As drug industry struggles, chemists face layoff wave," The Wall Street Journal, 11 December 2007, A1).

Chemical biologists have much to offer the pharmaceutical industry as it grapples with its current challenges. Two ongoing priorities for expanding the drug pipeline are identifying new biological targets and providing rapid access to lead compounds with enhanced potential for success in the clinic. Chemical biologists can support these efforts by providing new mechanistic insight into how drug candidates act in biological systems and by rigorous validation of biological targets. Second, chemical biology provides ways to expand and search chemical space for new bioactive compounds (Nat. Chem. Biol. 3, 433, 2007). Though these processes already occur in pharmaceutical research, the contributions of chemical biologists will only strengthen these efforts (for a Perspective, see Nat. Chem. Biol. 2, 689-700, 2006). Chemical biology will continue to make distinct contributions by pioneering new methods that could significantly change the way drug discovery is performed (Nat. Chem. Biol. 3, 187-191, 2007). For example, in the current issue, Krutzik et al. (p. 132) report a new drug screening platform based on flow cytometry that is designed to achieve parallel and real-time measurement of smallmolecule effects on multiple signaling networks within whole diseaserelevant cells. In addition to being valuable for identifying viable drug leads, the approach offers a powerful tool for monitoring therapeutic responses along the drug development pathway.

What can chemical biologists and pharmaceutical researchers do to foster synergy between their disciplines? Many answers to this question emerged at a recent conference entitled "Drug Action and Chemical Biology in the Postgenomic Era" (Nat. Chem. Biol. 4, 7-11, 2008). Both communities share a desire to understand the roles and actions of small molecules in cells and organisms. As a result, one way to increase the efficiency and success of drug discovery efforts is to examine the mechanisms of drug candidates in a more global, physiological context much earlier in the drug discovery process. This may be achieved in part by broader integration of new technologies that are emerging from academic chemical biology laboratories and small companies. Given the shared research interests of the two communities, the best and simplest mechanism for enhancing synergy between chemical biologists and pharmaceutical researchers is likely to be greater openness and expanded collaboration. These ongoing interactions offer essential opportunities for academic and industrial leaders to establish a scientific agenda that will shape the future of drug discovery research. 